\title{
Sentence comprehension and relative pronouns ${ }^{1}$
}

DAVID T. HAKES ${ }^{2}$ AND HELEN S. CAIRNS THE UNIVERSITY OF TEXAS AT AUSTIN

\begin{abstract}
Doubly self-embedded sentences, differing only in whether the relative pronouns were present or deleted, were presented to two groups of $S s$ who were required to respond to a word beginning with a particular letter in each sentence and to paraphrase the sentence. The results of both the phoneme monitor task and the paraphrase task indicated that comprehension was better when the relative pronouns were present than when they were deleted. These results are consistent with earlier results for the paraphrase task but are not consistent with earlier results for the phoneme monitor task. Possible reasons for the inconsistency were considered. It was concluded that the phoneme monitor task does reflect comprehension difficulty in a manner consistent with the paraphrase task and that deleting the relative pronouns does make comprehending self-embedded sentences more difficult.
\end{abstract}

Fodor and Garrett (1967; see also Fodor, Garrett, \& Bever, 1968) have recently presented a theory of sentence comprehension in which the rules of generative grammar are not directly represented in the process of comprehension. The theory assumes that comprehension involves at least the recovery of the grammatical relations underlying the sentence. In recovering these relations, the hearer (or reader) is assumed to utilize cues in the sentence's surface structure as a basis for projecting hypotheses about the underlying grammatical relations. How easy or difficult a sentence is to comprehend is predicted to be a function of the extent to which such surface cues are available, and further of the extent to which the grammatical relations receive a distorted representation in the surface structure. That is, where the grammatical relations are distorted or unmarked the sentence should be more difficult to comprehend than where they are undistorted and marked.

The experiments that Fodor and Garrett first presented in support of this theory involved doubly self-embedded (SE) sentences like (1) where the relative pronouns were present and (2) where they were deleted. (1) and

(1) The car which the man that the dog bit drove crashed.
(2) The car the man the dog bit drove crashed.

(2) will be referred to as unreduced and reduced, respectively. The presence of the relative pronouns was hypothesized to provide important cues indicating that sentences had been embedded within other sentences as relative clauses. While the same information is available in the reduced SE sentences from other cues (e.g., the occurrence of three successive noun phrases followed by three successive verb phrases), the pronouns were hypothesized to make this information available more readily and earlier and, hence, to make comprehension easier and quicker.

In Fodor and Garrett's experiments, Ss heard nine doubly SE sentences, either reduced or unreduced. After hearing a sentence, $S$ was to paraphrase it, i.e., to say in his own words what it meant. Each sentence was presented and paraphrased five times in succession. The data were analyzed in terms of paraphrase accuracy and also in terms of response delay (i.e., the latency of the paraphrase). Performance was better for the unreduced setences for both response measures. That is, the paraphrases were both quicker and more accurate when the sentences were unreduced.

More recently, however, Foss and Lynch (1969, Experiment II) have reported an experiment whose results appear inconsistent with those of Fodor and Garrett. The Foss-Lynch experiment compared right-branching (RB) sentences like (3) with both reduced and unreduced doubly SE sentences.

(3) The dog bit the man that drove the car which crashed.

Ss were instructed to listen in each sentence for a word beginning with a particular letter (always a b) and, if they heard one, to press a button. Response latency on this 'phoneme monitor' task was taken as a measure of comprehension difficulty. Foss and Lynch assumed that the processing of different kinds of information in a sentence (e.g., syntactic and phonemic) is not independent. Thus, when processing the syntactic information is difficult, there should be less processing capacity available for dealing with phonemic information. Hence, when the syntax is difficult to process, the response latency to a phonemic event should be longer than when it is not.
On the well-supported assumption that RB sentences are easier to comprehend than SE sentences, Foss and Lynch predicted that response latency in the phoneme monitor task would be less for RB than for SE sentences when the monitor word (i.e., the word containing an initial b) occurred late in the sentence. They predicted no difference between RB and SE sentences when the monitor word occurrred early since, up to the point where it occurred, the syntactic complexity of the two constructions was the same. Moreover, on the basis of Fodor and Garrett's results, they predicted that for late-occurring monitor words there would be a latency difference between reduced and unreduced SE sentences, the latter being responded to faster.

Foss and Lynch presented their Ss 90 sentences, $30 \mathrm{RB}, 30$ reduced $\mathrm{SE}$, and 30 unreduced SE. For each type, the monitor word was the first noun for 10 , the last verb for 10, and did not occur at all for 10 . Their results indicated that there were no sentence type differences when the monitor word occurred early. When it occurred late, latency for the RB sentences was significantly less than for either SE type. But there was no difference between reduced and unreduced SE sentences.

If the phoneme monitor task and the paraphrase task are both taken as reflecting comprehension difficulty, this last result is inconsistent with Fodor and Garrett's hypothesis and with their data. And it seems intuitively plausible that both tasks do reflect comprehension difficulty. Further, there is considerable motivation for determining whether or not they do, for there is a notorious problem with the paraphrase task. This task reflects only failures of comprehension. The fact that people have relatively little difficulty in understanding sentences except when they are inordinately complex leads to the problem with the task. Unless the sentences are so complex as to yield high rates of paraphrase errors, there is little chance that differences in difficulty between sentences will be reflected in the results. It is this insensitivity of the paraphrase task that has led experimenters to use such exotic sentences as double self-embeddings.

Given the inadequacies of the paraphrase task, it would be highly desirable to find some task more sensitive to differences in 
comprehension difficulty and, particularly, sensitive to differences between sentences that are not so difficult as to be incomprehensible. But while Foss's phoneme monitor task seems a possible candidate, there remains the task of accounting for the failure of this task to yield the same difference between reduced and unreduced $S E$ sentences as the paraphrase task. Thus the problem is not just one of accounting for the apparent empirical discrepancy betwen Fodor and Garrett's results and those of Foss and Lynch. More important, it is one of determining the comparability of the phoneme monitor and paraphrase tasks.

The present experiment was undertaken to explore this problem. Specifically, a direct comparison of the two tasks was undertaken by collecting both paraphrase and monitoring latency data from the same Ss and using the same SE sentences, reduced and unreduced.

\section{Materials}

\section{METHOD}

Eight of the nine sentences used by Fodor and Garrett were used. ${ }^{3}$ In addition, 12 filler sentences were used which were structurally complex but did not involve double selfembedding. The 20 sentences were ordered randomly with the restriction that no more than two of the SE test sentences appear in succession. The letter chosen for monitoring in each sentence was always the first letter of a word and did not appear as the first letter of any other word in that sentence. For seven of the eight SE test sentences the monitor word was the second verb. For the eighth it was the first verb since the first letter of the second verb also occurred as the first letter of another word. For eight of the filler sentences the monitor words were located in varying positions and represented a variety of form classes. The monitor words tended to occur earlier in the filler sentences than in the SE test sentences to prevent $S s$ from forming a set for late-occurring monitor words. For the remaining four filler sentences the letters for which Ss were to monitor did not occur in word-initial position. This was to prevent $S s$ from becoming set to always find a monitor word.

Two tapes were recorded, which included instructions about the tasks and about what letter to listen for in each sentence and the sentences. The tapes differed only in that the relative pronouns were deleted from the SE test sentences on one (Group 1) and were present on the other (Group 2). The sentences were recorded in normal sentence intonation by a female native speaker of English. The materials were recorded on a single track but were presented binaurally. Pulses were recorded on a different track of the tape, coincident with the beginning of each monitor word. These werc used to start a timer for measuring monitoring latency.

\section{Procedure}

When each $S$ appeared at the laboratory he was seated in front of a microphone and given headphones. The recorded instructions were then presented. The Ss were told that before hearing each sentence they would be told to listen for a word beginning with a particular letter (e.g., "Listen for a $k$ as in king."). If they heard a word beginning with that letter they were to press a hand-held button as quickly as possible. They were also told that as soon as each sentence was finished they were to say in their own words what it meant. Four practice sentences were then presented that were structurally complex but that did not involve double self-embedding. Three of the practice sentences contained monitor words while the fourth did not. Following presentation of the practice sentences, questions were answered.

The sentences were then presented. Following the presentation of each sentence, the tape recorder was stopped, and $S$ gave his paraphrase. He was allowed as much time as needed for paraphrasing, and the next sentence was presented approximately 3 sec after he had finished. The entire experimental session was recorded for later analysis of the paraphrase data.

\section{Subjects}

Twenty Ss served in each of the two groups and were assigned to groups randomly in order of appearance. All served as part of a requirement for the introductory psychology course. Five Ss were discarded, four for failure to follow instructions and one because he was not a native speaker of English. Each was replaced by the next $S$ to appear. Two Es each tested half the Ss in each group.

\section{RESULTS AND DISCUSSION \\ Paraphrase Task}

The paraphrase data for the SE sentences were transcribed from the tapes made during the experiment. ${ }^{4}$ The data were scored, independently and without knowledge of Ss' group membership, by two scorers using the criteria developed by Fodor and Garrett (i.e., in terms of the number of propositions from each sentence that were represented accurately in S's paraphrase). ${ }^{5}$

A paraphrase accuracy score for each $S$ was computed by summing the number of propositions accurately paraphrased across the eight SE sentences. The mean accuracy for Groups 1 (reduced) and 2 (unreduced), respectively, were 11.50 and 13.85 out of a possible 24.00. These scores are not directly comparable to those reported by Fodor and Garrett for the same sentences. Their data are presented in terms of paraphrase accuracy over five trials per sentence weighted by response delay.

The accuracy scores were subjected to a 2 by 2 , Groups by Experimenters, analysis of variance. The main effect for groups yielded $F(1,36)=5.37, p<.05$. The main effect of experimenters and the interaction both yielded $F_{s}<1$. The results are thus consistent with those obtained by Fodor and Garrett-paraphrasing is more accurate when the relative pronouns are present than when they are deleted.

The effect of relative pronoun deletion is quite consistent across sentences. Seven of the eight SE sentences yielded higher accuracy scores for Group 2 than for Group 1. No statistical analysis was performed on the sentences effects, however, as none would have been orthogonal to that reported above for groups. It is interesting to note that in three of the five Fodor and Garrett experiments there was also one sentence for which the predicted effect did not occur. The particular sentence was different in each of their three cases, and it was different here from any of their cases. This would suggest that the effect of relative pronoun deletion is quite general. The occasions on which individual sentences failed to yield the effect are probably best treated as chance deviations.

The paraphrase data were also scored in terms of response delay, i.e., the length of the interval between the end of the presented sentence and the beginning of a paraphrase response that $S$ himself accepted. The delays were obtained by running the paraphrase tapes through a high-speed graphic level recorder and marking the relevant distances. These distances were converted into seconds of response delay, and the delays were in turn converted by reciprocal transformation into speed scores. A mean speed score for the eight SE sentences was then computed for each $S$. The group means of these mean ipeed scores for Groups 1 and 2 , espectively, were $0.301 / \mathrm{sec}$ and $0.386 / \mathrm{sec}$. (Note that a higher speed score corresponds to a shorter latency, and, hence, to a faster response.)

The mean speed scores were subjected to a 2 by 2 analysis of variance. No significant differences were found, the effect for groups yielding $F(1,36)=2.17, p>.05$, and the effects for experimenter and the interaction both yielding $\mathrm{Fs}<1$. Thus the results for response delay, although in the predicted direction, are not entirely consistent with the other results of the 
present experiment or with the response delay results obtained by Fodor and Garrett. The effect does seem to consistent across sentences, seven the eight yielding differences in the pr. ad direction. This suggests that the re. delay effect may be real, but smi! and unreliable.

\section{Monitor Task}

Response latencies on the monitor tas. were converted to speed scores, and all analyses were performed on these scores. Two scores were computed for each S: mean speed on the $S E$ sentences and mean speed on the filler sentences. The speed scores for the filler sentences were subjected to a 2 by 2 analysis of variance. Both main effects yielded $F_{s}<1$, and the interaction was also not significant, $F(1,36)=1.97, p>.05$. There is thus no evidence of differential subject or experimenter effects.

The mean speed scores for the SE sentences were $1.68 / \mathrm{sec}$ and $2.18 / \mathrm{sec}$ for Groups 1 and 2, respectively. These speed scores are considerably faster than those for the most closely comparable conditions of the Foss-Lynch experiment-1.60/sec and $1.47 / \mathrm{sec}^{6}$ (These are the Foss-Lynch means for reduced and unreduced doubly SE sentences where the monitor word was the last verb.) The speed scores were subjected to a 2 by 2 analysis of variance. The main effect for groups yielded $F(1,36)=10.58, p<.01$. The main effect for experimenters and the interaction both yielded Fs $<1$.

It would appear, then, that in the present experiment the monitor task yielded results consistent with those of the paraphrase task and also consistent with Fodor and Garrett's hypothesis and their results for the paraphrase task. They are not consistent, however, with the results by Foss and Lynch for the monitor task. We will return to consider this inconsistency later.

Here, as for the paraphrase task, the effect of relative pronoun deletion is consistent across sentences. Seven of the eight sentences yielded faster mean speed scores for Group 2 than for Group 1. Again, the exceptional sentence was a different one from the exceptions in any of the results for paraphrasing.

In general, then, the results of the present experiment are consistent with the prediction made by Fodor and Garrett and with their results. Most significantly, the present results indicate that the effect of relative pronoun deletion can be obtained using the phoneme monitor task. The data give us no cause to reject the claim that $S$ s make use of surface structure cues, when available, for determining the underlying grammatical relations of a sentence. There are, however, a number of points about the present and previous data that require comment.

First, it should be noted that in the present experiment the paraphrase task yielded the predicted effect for accuracy even though Ss were given only a single opportunity to paraphrase each sentence. The effect obtained by Fodor and Garrett can, therefore, not be attributed to the fact that their Ss paraphrased each sentence five times in succession. It cannot be the case, that is, that the effect appeared only because their Ss had repeated opportunities to paraphrase.

The response delay data also deserve comment. The fact that Fodor and Garrett obtained significant effects for this measure while we did not is partly attributable to their averaging data across five trials for each senterice. Response delays here (i.e., for a single trial) were highly variable across both sentences and Ss. It seems likely that with repeated paraphrases this variability would decrease.

It should also be noted that there are inherent difficulties in the use of this measure to reflect comprehension difficulty. Some Ss, for example, consistently emitted the first several words of a paraphrase (e.g., a noun phrase) quite quickly and then paused for several seconds before continuing. Since they did not repeat or change what they had started to say, the paraphrase was counted as having started when they first spoke. Other Ss delayed before starting and then completed the paraphrase without hesitation while still others started, stopped, and then either repeated or changed with they had said. Such differences suggest that it is a rather dubious procedure to treat response delay measured to the beginning of an uncorrected paraphrase, as a measure of the time taken to comprehend the sentence.

It would perhaps seem more reasonable to use the total time taken to paraphrase, i.e., to measure to the end rather than to the beginning of the paraphrase. However, this measure is also contaminated, for total time is correlated with length of paraphrase, and the more adequate paraphrase is likely to be longer than the less adequate. For these reasons, and others as well, we suggest that response delay has little to recommend it as a measure of comprehension difficulty.

The problem that we have intentionally left until last is that of accounting for the difference between the present results and those of Foss and Lynch for the phoneme monitor task. There are, of course, a number of obvious differences between the experiments, any of which might account for the difference in results. All of Foss and Lynch's sentences were either right-branching or self-embedded while those used here included structurally heterogeneous sentences as fillers. One might suppose that the high proportion of SE sentences ( $2 / 3 \mathrm{rds})$ in their experiment might have led Ss to adopt some strategy that eliminated the difference between reduced and unreduced SE sentences. Such an account seems implausible inasmuch as the difference was obtained by Fodor and Garrett, albeit with the paraphrase task, in experiments where all the sentences were self-embedded.

The length of the Foss-Lynch experiment might also have contributed to their results. They presented each $S 90$ sentences, using a procedure where there was a fixed interval of $30 \mathrm{sec}$ between the end of one sentence and the beginning of the next. The S's task during this interval was a fill-in-the-blanks comprehension test that rarely took more than $10 \mathrm{sec}$, leaving long 'dead' intervals between sentences. One might suppose that this, combined with the overall length of the experiment $(90 \mathrm{~min})$, might have resulted in boredom or some other effect that masked the relative pronoun effect. However, it would be difficult to reconcile this account with the significant differences that Foss and Lynch did obtain. Further, inspection of their data suggests that performance did not change materially from the beginning to the end of the experiment.

There is, of course, the possibility that the difference in results is attributable to the particular sentences used, i.e., that the effect is sentence-specific. The fact that the effect is quite consistent across sentences, both here and in the Fodor and Garrett experiments, suggests that this is an unlikely possibility.

There is, however, a materials problem internal to the Foss-Lynch experiment that might be related to its outcome. The SE sentences without relative pronouns were the same for all $S s$ and always different from those with relative pronouns. Although the sentences that appeared in reduced and unreduced form were selected randomly from a single set of sentences, it is conceivable that the two subsets differed systematically in some way.

A number of other small procedural differences between the two experiments deserve passing mention. Foss and Lynch's Ss monitored for the same letter throughout while for our Ss the letter changed with every sentence. The comprehension test was in one case a fill-in-the-blanks task and in the other the paraphrase task. And, finally, in the Foss-Lynch experiment the monitor word was the last verb while here it was the 
second (and in one case, the first) verb. Like the rest of the differences we have mentioned, there is no readily apparent reason why any of these should have produced the difference in results.

We are left, then, with no account of the difference between the present results and those obtained by Foss and Lynch. What does seem clear is that the phoneme monitor task, like the paraphrase task, can reflect differences in comprehension difficulty.

\section{REFERENCES}

FODOR, J. A., \& GARRETT, M. Some syntactic determinants of sentential complexity. Perception \& Psychophysics, 1967, 2, 289-296.

FODOR, J. A., GARRETT, M., \& BEVER, T.G. Some syntactic determinants of sentential complexity, II: Verb structure. Perception \& Psychophysics, 1968, 3, 453-461.
FOSS, D. J., \& LYNCH, R. H., Jr. Decision processes during sentence comprehension: Effects of surface structure on decision times. Perception \& Psychophysics, 1969, 5, 145-148.

\section{NOTES}

1. This research was supported in part by NSF Grant GB-3629 to the senior author and Robert K. Young and in part by NDEA Title IV Fellowship No. 67-11070 to the junior author. The authors wish to express their thanks to Barbara Tabor for her assistance in collecting and anaiyzing the data and to Donald J. Foss and Merrill Garrett for their cooperation and comments. Portions of this research were reported to the Midwestern Psychological Association Meetings, Chicago, May 1969.

2. Department of Psychology, The University of Texas at Austin, Austin, Tex. 78712.

3. The omitted sentence was also omitted by Fodor and Garrett from all of their analyses on the ground that their Ss found it meaningless. 4. Paraphrase data for the filler sentences were not analyzed, in part because of the amount of effort that would have been involved in transcribing them and in part because the scoring criteria for the $S E$ sentences could not have been applied to them in a straightforward manner.

5. See Fodor, Garrett, and Bever (1968) for a discussion of the scoring criteria. To insure that the scoring here would be comparable to that in the Fodor-Garrett experiments, Merrill Garrett kindly served as one of the scorers for the present experiment. The authors acknowledge his assistance with gratitude. The two scorers disagreed on the scoring of $2.1 \%$ of the propositions; these disagreements were resolved by discussion.

6. These speed scores cannot be obtained from the latency data reported by Foss and Lynch (1969). They were computed from the original data of that experiment. We thank Donald $J$. Foss for making these data available to us.

(Accepted for publication October 20, 1969.) 